\title{
A TECHNIQUE FOR MONITORING FAST TUNER PIEZOACTUATOR PRELOAD FORCES FOR SUPERCONDUCTING RF CAVITIES*
}

\author{
Y. Pischalnikov\#, J. Branlard, R. Carcagno, B. Chase, H. Edwards, D. Orris, A. Makulski, M. \\ McGee, R. Nehring, V.Poloubotko, C. Sylvester, S. Tariq, FNAL, Batavia, IL 60510, U.S.A.
}

\begin{abstract}
The technology for mechanically compensating Lorentz Force detuning in superconducting RF cavities has already been developed at DESY. One technique is based on commercial piezoelectric actuators and was successfully demonstrated on TESLA cavities [1]. Piezo actuators for fast tuners can operate in a frequency range up to several $\mathrm{kHz}$; however, it is very important to maintain a constant static force (preload) on the piezo actuator in the range of 10 to $50 \%$ of its specified blocking force. Determining the preload force during cool-down, warm-up, or re-tuning of the cavity is difficult without instrumentation, and exceeding the specified range can permanently damage the piezo stack. A technique based on strain gauge technology for superconducting magnets has been applied to fast tuners for monitoring the preload on the piezoelectric assembly. The design and testing of piezo actuator preload sensor technology is discussed. Results from measurements of preload sensors installed on the tuner of the Capture Cavity II (CCII)[2] tested at FNAL are presented. These results include measurements during cool-down, warmup, and cavity tuning along with dynamic Lorentz force compensation.
\end{abstract}

\section{INTRODUCTION}

The lifetime and performance of a piezoelectric actuator is dependent on its preload [3]. For this reason, it is important to have reliable techniques for monitoring piezo actuator preload forces with respect to different operational conditions over a wide range of temperatures ( $2 \mathrm{~K}$ to $300 \mathrm{~K}$ ).

Piezo preload can be determined by monitoring operational characteristics; such as actuator capacitance or impedance resonance shifts [4]. A technique developed at FNAL exploits the calibrated stress and strain response of a stainless steel (SS) element instrumented with strain gauges and inserted in-line with the piezo stack. Results of these measurements are presented in this paper.

\section{SINGLE PIEZO FAST TUNER}

\section{Tuner Fixture Design}

FNAL's CCII Fast Tuner Fixture (FTF) design is a modification of the original DESY single piezo tuner assembly. The modifications include the addition of diagnostic instrumentation such as the SS "bullet" strain

*Work supported by U.S. Department of Energy under contract DEAC02-76CH03000

\#pischaln@fnal.gov gauge device. The shape of this sensing device is a cube $10 \times 10 \times 10 \mathrm{~mm}^{3}$ with a half sphere machined on the bottom to provide ball-joint-like motion to reduce shear forces. The top end of the bullet is flat in order to mate against the piezo stack (PI Ceramic P-888.91). Four strain gauges (Micro Measurements Vishay WK-06-062AP-350W) have been attached to the sides of bullet with cryogenic epoxy (M-BOND 610). See Fig. 1.

Cernox ${ }^{\circledR}$ RTD's have been mounted on the FTF's SS bracket and on the piezo stack to provide temperature data. An additional strain ("compensating”) gauge (CSG) has been attached to the surface of the bottom bracket. Copper-beryllium (CuBe2) disc springs have provided a constant preload force to the piezo stack.

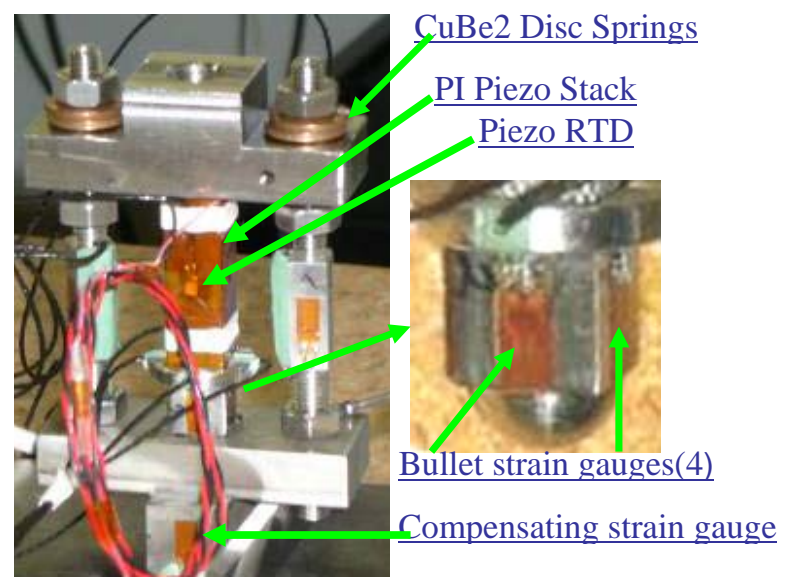

Figure 1: CCII Fast Tuner Fixture. Insert: picture of Bullet with 4 strain gauges.

\section{Bullet Calibration}

Forces applied to the bullet sensor will result in a measurable stress, which can be determined by the change in resistance of the strain gauges glued to the four sides of the bullet sensor. Equation (1) is used to calculate the forces applied to the bullet:

$$
F(T)=\frac{1}{4} \sum_{i=1}^{4}\left\{K_{i}(T) *\left[R_{i}(T)-R_{i}^{0}(T)\right]\right\}
$$

where $i=1 . .4$ is strain gauge number; $K_{i}(T)$ is a calibration coefficient, $R_{i}(T)$ is the measured resistance of the strain gauge when a force $\mathrm{F}$ is applied to the bullet, and $R_{i}^{0}(T)$ is the resistance of the strain gauge when no 
force is applied. The calibration coefficient $K_{i}(T)$ was determined by applying a control force on the bullet while measuring the resistance changes of the strain gauges (Fig. 2). For the calibration at $\mathrm{T}=290 \mathrm{~K}$, the piezo stack on the FTF (Fig.1) was replaced by a small size load cell. A control force was applied to the load cell and bullet by tightening nuts above disc springs. The resulting calibration coefficient measured by this method averaged over the four strain gauges was: $K_{i}(T=290 K)=$ $2.6 * 10^{4} \mathrm{~N} / \mathrm{Ohm}$.

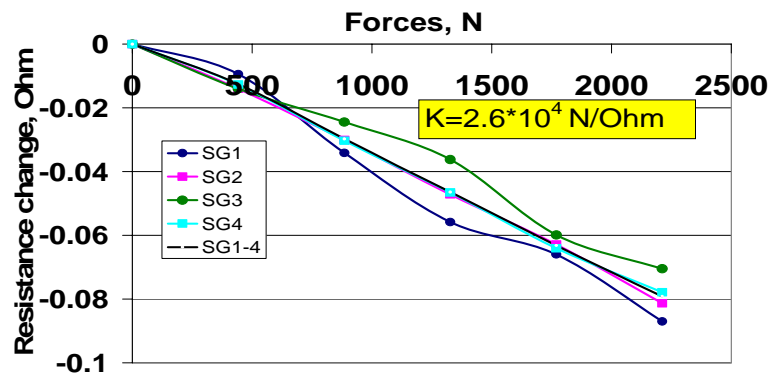

Figure 2: Calibration of bullet's SGs at $\mathrm{T}=290 \mathrm{~K}$.

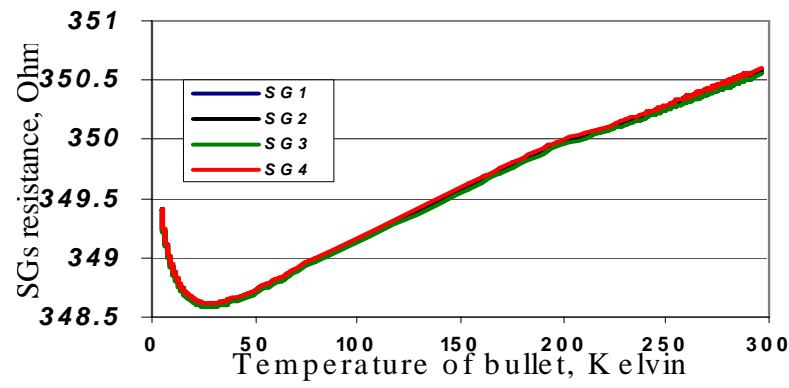

Figure 3: Measurements SGs resistance for unloaded bullet $(\mathrm{F}=0)$ versus temperature.

Calibration of the bullet sensors at $\mathrm{T}=4 \mathrm{~K}$ was done in the FNAL/Technical Division calibration facility. This facility is capable of calibrating different types of force sensors instrumented with strain gauges from room temperature down to $\mathrm{T}<4 \mathrm{~K}$. For the bullet sensor calibration, the calibration coefficient was determined to be $10 \%$ lower at $300 \mathrm{~K}$ than at $4 \mathrm{~K}$, which was expected. This is a direct result of differences in modulus of elasticity for stainless steel at different temperatures.

The size of the "bullet" does not allow the strain gauges to be configured in a Wheatstone bridge, so $R_{i}^{0}(T)$ has been measured as a function of temperature by thermocycling the unloaded bullet from $\mathrm{T}=290$ to $\mathrm{T}=4 \mathrm{~K}$ (Fig.3). An additional, unloaded strain gauge (CSG) was also used to compensate for $R_{i}^{0}$ changes with temperature. The CSG is attached to a part of the fixture which experiences no stress or strain but which is in close thermal contact with the bullet.

\section{RESULTS}

\section{Monitoring Piezo Preload.}

The results from monitoring the piezo preload using the bullet sensor instrumentation when CCII was thermallycycled are shown in Fig. 4. The Fast Tuner Fixture is located in an insulated vacuum space and, as a result the temperature of the piezo stack was never below 20K even when the cavity was cooled down to $1.8 \mathrm{~K}$. The following is a summary of the results presented in Fig.4:

- The piezo preload, measured by averaging the reading from all four bullet SGs, increased from $2500 \mathrm{~N}$ at $290 \mathrm{~K}$ up to almost $5000 \mathrm{~N}$ ( $75 \%$ of blocking force for PI P-888.91) at $\mathrm{T}<20 \mathrm{~K}$.

- The piezo stack preload returns to $\sim 2500 \mathrm{~N}$ at the end of a thermal-cycle.

- Each of the four SGs experienced different stress changes during the thermal-cycle. This data leads to the conclusion that the Fast Tuner assembly was bending and twisting during the cool down. In order to estimate the shearing forces on the piezo stack, we have used data (from Fig. 4) as input for an ANSYS simulation. The results from this calculation determined that shearing forces of $\sim 500 \mathrm{~N}$ were developed.

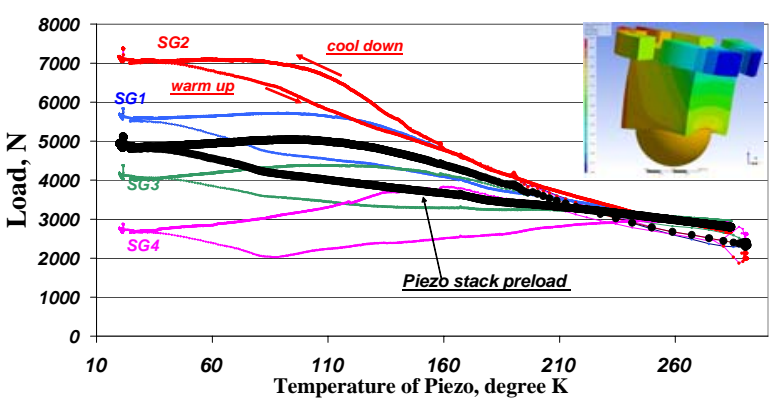

Figure 4: Piezo actuator preload as a function of piezo temperature during CCII thermocycle. Preload, measured independently by each of four SGs, and calculated average (black). Insert: results of ANSYS simulation, when $2500 \mathrm{~N}$ axial and $500 \mathrm{~N}$ shearing forces are applied to the bullet.

The additional preload on the piezo stack at $4 \mathrm{~K}$ could be explained by a difference $(\delta \mathrm{X} \sim 60 \mu \mathrm{m})$ between the thermal-contraction of the tuner SS frame and the piezo stack. The spring constant $\left(\mathrm{K}_{\mathrm{ds}}=45 \mathrm{~N} / \mu \mathrm{m}\right)$ of the discs springs used in the FTF (Fig. 1) has been measured at $4 \mathrm{~K}$. As a result, it is estimated that the extra load on the piezo during cool down is $\sim 2700 \mathrm{~N}$. A simple way to avoid excessive preload increase during cool down is to use a weaker disc spring for FTF.

Development of quite large ( $500 \mathrm{~N})$ shearing forces on the piezo is a results of FTF twisting during cool-down, coupled with a large coefficient of friction between bullet and its nesting position at the bracket, both of which are made of stainless steel. 
As reported by several researchers, quite often Fast Tuners fail, when attempts are made to tune the SCRF cavity to the nominal $(1.3 \mathrm{GHz})$ resonance frequency after

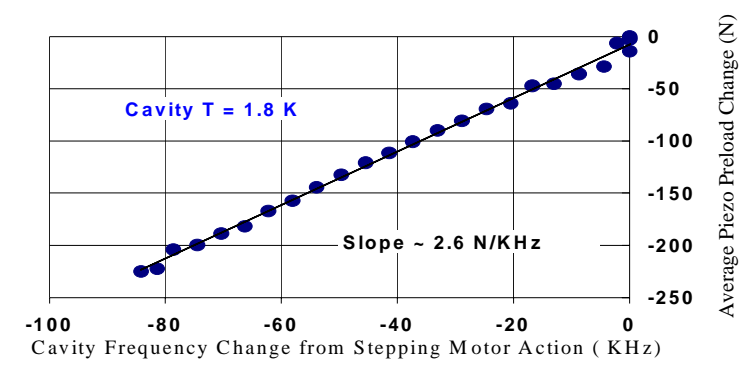

Figure 5: Change of Fast Tuner preload as the cavity was retuned by $85 \mathrm{kHz}$ using the slow tuner.

cavity cool-down to cryogenic temperature. The retuning range of a SCRF cavity by the Slow Tuner could be up to several hundreds of $\mathrm{KHz}$. The mechanical linkage between Slow and Fast Tuners will lead to a piezo stack preload change. Results of monitoring piezo preload with bullet's SGs instrumentation, when CCII was been retuned by $\Delta \mathrm{F}=-85 \mathrm{kHz}$, are presented in Fig. 5. A linear fit to the data provides a coefficient for piezo preload changes of $\sim 2.6 \mathrm{~N} / \mathrm{kHz}$. This measured value is quite close to the coefficient calculated from a mechanical model of cavity.

\section{Fast Tuner Performance.}

The CCII Fast Piezo Tuner performs well despite the increased preload and the shearing forces that developed during cool-down. Figs. 6 and 7 show the Lorentz force detuning of CCII at $26 \mathrm{MV} / \mathrm{m}$ with and without fast tuner compensation. Driving the tuner with a train of four 180

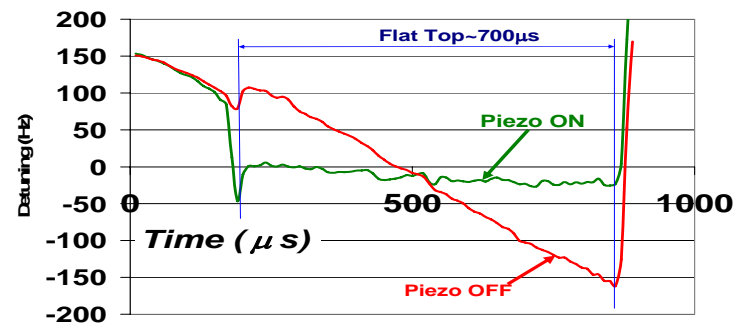

Figure 6: CCII resonance frequency shift induced by Lorentz force, when $E_{a c c}=26 \mathrm{MV} / \mathrm{m}$ (red line). The green line shows the CCII detuning when the Fast Tuner is ON.

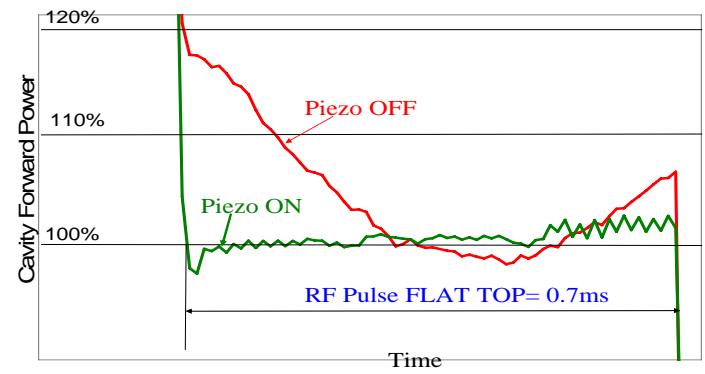

Figure 7: Forward RF power during CCII closed loop "flat top" at $E_{\text {Acc }}=26 \mathrm{MV} / \mathrm{m}$. The red line shows forward power signal when Fast Tuner is OFF and the green line when Piezo is ON.
$\mathrm{Hz}$ sinusoidal cycles of amplitude $\mathrm{A}_{\mathrm{Sin}}=22 \mathrm{~V}$, reduced the cavity detuning from $\sim 275 \mathrm{~Hz}$ to $\sim 20 \mathrm{~Hz}$ (Fig. 6). At the same time Fast Tuner reduced the forward power required to run the cavity by $\sim 20 \%$ (Fig. 7) at $\mathrm{Q}_{\text {Loaded }}$ of $2 \cdot 10^{6}$.

Fig. 8 shows the data from measurements of CCII microphonics (cryo-flow induced mechanical vibrations of the cavity) while the cavity operated in Continuous Wave (CW) mode with $10 \mathrm{~W}$ forward power. The "red" line is the signal from the piezoelectric stack, functioning as a sensor. The "blue" line is a signal from the phase detector, which measures the phase between forward power and transmitted power

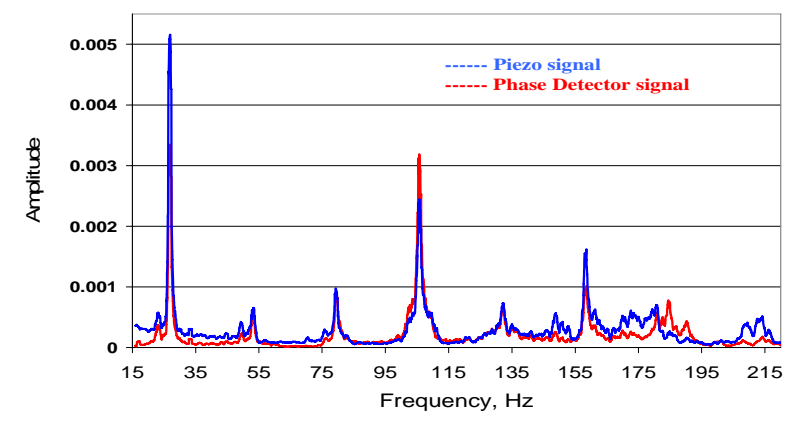

Figure 8: Frequency spectrum of cryo-flow induced vibrations in CCII.

\section{SUMMARY}

Strain gauge technology has been used successfully to instrument the CCII Fast Piezoelectric Tuner. The gauges monitored piezo actuator preload and shear during cavity thermo-cycles and during retuning of the cavity with the slow tuner. The excess preload $(\sim 2500 \mathrm{~N})$ and the shearing forces $(\sim 500 \mathrm{~N})$ that developed during cool down of CCII did not compromise performance of the piezo tuner as an actuator [5] or as a sensor. The Fast Tuner reduced Lorentz force detuning from $\sim 275 \mathrm{~Hz}$ to $\sim 20 \mathrm{~Hz}$ during CCII operations at $26 \mathrm{MV} / \mathrm{m}$.

\section{REFERENCES}

[1] M. Liepe, et al “ Dynamic Lorentz Force Compensation with Fast Piezoelectric Tuner", PAC2001, Chicago, USA, pp 1074-1076

[2] T. W. Koeth, et al "Capture Cavity II at Fermilab" LINAC2006, Knoxville, Tennessee, USA, pp719-721

[3] Zickgraf, et al "Fatigue Behavior of Multilayer Piezoelectric Actuators", Application of Ferroelectrics, 1994. ISAF' 94, pp. 325-328

[4] P.Sekalski, et al "Static Absolute Force Measurements For Preloaded Piezoelements Used For Active Lorentz Force Detuning System” LINAC 2004, Lubeck, Germany, pp. 486-488

[5] M. W. McGee and Y. Pischalnikov "Mechanical Stability Study of Capture Cavity II at Fermilab" PAC'07, also in these proceedings, 2007 
